\title{
Pathogenesis and treatment of intracranial arachnoid cysts in pediatric patients younger than 2 years of age
}

\author{
Gabriel Zada, M.D., Mark D. Krieger, M.D., Sean A. MCNatt, M.D., Ira Bowen, B.S., \\ AND J. GORDON MCCOMB, M.D.
}

Department of Neurosurgery, Childrens Hospital Los Angeles, Keck School of Medicine of University of Southern California, Los Angeles, California

\begin{abstract}
Object. Arachnoid cysts can cause a variety of clinical signs and symptoms in infants. The authors sought to determine whether the clinical presentation of pediatric patients younger than 2 years old and harboring arachnoid cysts influenced the type of intervention that would be required.

Methods. A retrospective chart review was conducted for all patients younger than 2 years of age who had undergone craniotomy for fenestration of an arachnoid cyst at the Childrens Hospital Los Angeles between 1995 and 2006.

Forty-two patients were included in the study. The mean age was 10.4 months. The median follow-up time was 33 months. Clinical presentations were as follows: macrocephaly without ventriculomegaly ( 21 patients, $50 \%$ ), hydrocephalus (six patients, 14\%), and other symptoms (15 patients, 36\%). After fenestration of the arachnoid cyst, 12 of 21 patients $(57 \%)$ presenting with nonspecific macrocephaly required placement of a cystoperitoneal or ventriculoperitoneal shunt, compared with 1 of 15 patients $(7 \%)$ presenting with other symptoms $(p$ value $=0.0039)$. Five of six patients with hydrocephalus (83\%) were shunt dependent following fenestration. Overall, 18 of 42 patients $(43 \%)$ were shunt dependent after fenestration. Ten of these patients $(55 \%)$ required revisions during the follow-up period.

Conclusions. Patients younger than 2 years of age and harboring an arachnoid cyst commonly present with macrocephaly. These patients are more likely to require shunts than are those presenting with other findings, such as seizures or incidental lesions. The development and expansion of arachnoid cysts may be related to aberrant cerebrospinal fluid dynamics, and these lesions may be a form fruste of hydrocephalus. Arachnoid cysts should be treated with craniotomy and cyst fenestration, taking into account the likelihood of perioperative shunt dependency.
\end{abstract}

\section{KEY WORDS • arachnoid cyst • hydrocephalus - macrocephaly • fenestration • cerebrospinal fluid $\bullet$ children}

A RACHNOID cysts are developmental anomalies occurring between layers of the arachnoid membrane that may become symptomatic via mass effect on surrounding structures, spontaneous rupture or hemorrhage, or obstruction of CSF outflow pathways. It seems likely that the development and growth of arachnoid cysts, in many cases, is related to the abnormal flow of CSF. Some authors have postulated a correlation between arachnoid cysts and hydrocephalus, yet the precise relationship between these entities remains to be fully elucidated. ${ }^{7,17,21}$ Furthermore, a direct correlation between macrocephaly without hydrocephalus and the development of arachnoid cysts may exist, also potentially suggesting an underlying aberrancy in CSF dynamics. ${ }^{15}$

There has been significant debate about the origins as well as the optimal surgical management of arachnoid cysts. ${ }^{3,6,7,13,21,25,28}$ The optimal treatment for lesions requiring surgical intervention, such as those causing symptoms or expanding (determined by sequential imaging), remains to be determined. Many institutions, such as ours,

Abbreviations used in this paper: $\mathrm{CP}=$ cystoperitoneal; $\mathrm{CSF}=$ cerebrospinal fluid; $\mathrm{CT}=$ computed tomography; $\mathrm{VP}=$ ventriculoperitoneal. have favored initial cyst fenestration, usually into the basal cisterns. ${ }^{4,7,8,11,13,14,16,21,23,25,28}$ Other centers have advocated the placement of $\mathrm{CP}$ shunts as a primary treatment for these cysts. ${ }^{3,27}$

The clinical presentation of patients with arachnoid cysts, especially during the first few years of life, may offer considerable insight into the relationship between these lesions and underlying aberrancies in CSF flow. This relationship has important implications for the type of surgical intervention that is likely to be successful. We conducted a retrospective analysis of a population of patients younger than 2 years of age who had undergone craniotomy for fenestration of an arachnoid cyst. Our goal was to further characterize the relationship between the clinical presentation of patients with an arachnoid cyst and the surgical intervention(s) required to successfully treat it, with particular attention to the requirements for shunt placement.

\section{Materials and Methods}

We conducted a search of the patient database in the Division of Neurosurgery at the Childrens Hospital Los Angeles for any patients who had undergone craniotomy with fenestration of an arachnoid cyst between the years 
1995 and 2006. Patients younger than 2 years old at the time of surgery were selected from this population and were included in the present study. Patient charts were retrospectively reviewed. Data from clinical notes, surgical reports, and radiographic reports were extracted and entered into a patient database. Data were subsequently analyzed in a confidential manner on approval from the institutional review board at Childrens Hospital Los Angeles and in compliance with Health Insurance Portability and Accountability Act regulations.

\section{Results}

\section{Study Population}

We identified and included in the study 42 patients who had undergone craniotomy for fenestration of an arachnoid cyst. The mean patient age was 10.4 months (range $0.2-23.9$ months). There were 25 boys (60\%) and 17 girls (40\%). The median follow-up period was 33 months.

Patients were classified into the following groups based on their initial clinical presentation (Table 1): macrocephaly (head circumference $>98$ th percentile) without ventriculomegaly (21 patients [50\%]), hydrocephalus (six patients [14\%]), other symptoms (15 patients [36\%]).

\section{Location of Arachnoid Cysts}

Arachnoid cysts were located in the following sites (Table 2): middle cranial fossa (22 patients [52\%]), posterior fossa (five patients $[12 \%]$ ), suprasellar region (five patients [12\%]), convexity/holohemispheric cysts (five patients [12\%]), interhemispheric cysts (four patients $[10 \%]$ ), and intraventricular cyst (one patient [2\%]).

Of the 22 patients presenting with middle fossa arachnoid cysts, three had concomitant ventriculomegaly. Two of these patients received shunts before undergoing cyst fenestration. Ten patients presented with middle fossa arachnoid cysts, normal ventricle size, and macrocephaly; six of these patients $(60 \%)$ required shunts postoperatively, five of which were CP shunts. Nine patients had neither macrocephaly nor ventriculomegaly; one of these patients $(11 \%)$ required VP shunt placement.

Five patients had posterior fossa arachnoid cysts; all had presented with macrocephaly, whereas only one had coexisting ventriculomegaly. Three of the patients underwent successful fenestration without subsequent shunt placement, whereas two required VP shunt placement after fenestration.

TABLE 1

Clinical presentation of 42 patients who underwent fenestration for an arachnoid cyst

\begin{tabular}{lc}
\hline \multicolumn{1}{c}{ Clinical Presentation } & No. of Patients $(\%)$ \\
\hline macrocephaly w/o ventriculomegaly & $21(50)$ \\
hydrocephalus & $6(14)$ \\
other symptoms & 15 \\
incidental finding & $6(14)$ \\
fetal ultrasonography & $4(10)$ \\
seizures & $2(5)$ \\
motor deficit & $1(2)$ \\
developmental delay & $1(2)$ \\
macrocephaly (<98th percentile) & $1(2)$ \\
total & $42(100)$ \\
\hline
\end{tabular}

TABLE 2

Relationship between clinical presentation and arachnoid cyst location

\begin{tabular}{|c|c|c|c|c|}
\hline \multirow[b]{2}{*}{$\begin{array}{c}\text { Cyst } \\
\text { Location }\end{array}$} & \multirow[b]{2}{*}{$\begin{array}{c}\text { No. of } \\
\text { Cysts }(\%)\end{array}$} & \multicolumn{3}{|c|}{ Clinical Presentation (No. of Patients) } \\
\hline & & $\begin{array}{l}\text { Hydro- } \\
\text { cephalus }\end{array}$ & $\begin{array}{l}\text { Macro- } \\
\text { cephaly }\end{array}$ & Other \\
\hline middle fossa & $22(52)$ & 3 & 10 & 9 \\
\hline posterior fossa & $5(12)$ & 1 & 3 & 1 \\
\hline suprasellar & $5(12)$ & 1 & 3 & 1 \\
\hline convexity & $5(12)$ & 0 & 3 & 2 \\
\hline interhemispheric & $4(10)$ & 1 & 2 & 1 \\
\hline intraventricular & $1(2)$ & 0 & 0 & 1 \\
\hline total $(\%)$ & $42(100)$ & (14) & $(50)$ & (33) \\
\hline
\end{tabular}

Five patients had suprasellar cysts. Three of them required placement of a VP shunt following fenestration, including the only one who had presented with hydrocephalus. Another of these patients underwent successful endoscopic fenestration with the creation of a third ventriculostomy.

Five patients had arachnoid cysts located along the convexities, primarily in the frontotemporal region. None of these patients presented with hydrocephalus. One of them required the subsequent placement of a CP shunt.

Four patients presented with interhemispheric cysts. One received a shunt before fenestration for hydrocephalus. Two others required CP shunts after fenestration.

\section{Patients Presenting With Macrocephaly}

Twenty-one patients (50\%), including 12 boys and nine girls, presented with macrocephaly (above the 98th percentile) without underlying ventriculomegaly. The mean patient age among this group was 10 months. None of these patients underwent placement of a shunt, either VP or CP, before fenestration of the arachnoid cyst. In the postoperative period, $12(57 \%)$ of 21 patients required CSF shunting of some type, whereas $43 \%$ remained shunt free (Table 3). Five patients underwent subsequent placement of a VP shunt after fenestration, whereas the other seven underwent postoperative placement of a CP shunt (Fig. 1). The mean time to shunt placement following craniotomy with fenestration was 1.8 months (median 1 month). Among the patients with available data, $40 \%$ demonstrated normalization of their head circumference

TABLE 3

Distribution of patients with arachnoid cysts requiring perioperative shunts, according to initial clinical presentation*

\begin{tabular}{lccccc}
\hline \hline & & & & \multicolumn{3}{c}{$\begin{array}{c}\text { Shunt Dependent } \\
\text { After Fenestration }\end{array}$} \\
\cline { 4 - 6 } $\begin{array}{c}\text { Clinical } \\
\text { Presentation }\end{array}$ & Total & $\begin{array}{c}\text { Before } \\
\text { Fenestration }\end{array}$ & Total & VPS & CPS \\
\hline macrocephaly & 21 & 0 & $12(57) \dagger$ & 5 & 7 \\
hydrocephalus & 6 & 3 & $5(83)$ & 5 & 0 \\
other & 15 & 0 & $1(7) \dagger$ & 1 & 0 \\
total & 42 & 3 & $18(43)$ & 11 & 7 \\
\hline
\end{tabular}

$*$ Values represent the number of patients $(\%)$. Abbreviations: $\mathrm{CPS}=\mathrm{CP}$ shunt; VPS = VP shunt.

$\dagger \mathrm{p}$ value $=0.0039$, two-tailed Fisher exact test. 


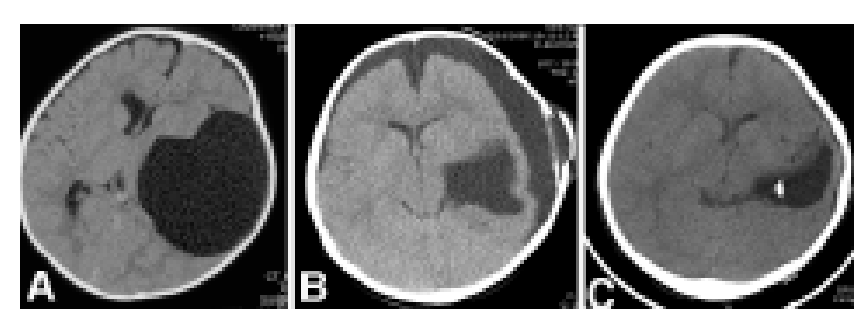

FIG. 1. A: Preoperative non-contrast-enhanced CT scan demonstrating a large left middle fossa arachnoid cyst. B: Initial followup CT scan showing a decrease in the cyst size and the development of subdural hygromas. C: Follow-up CT scan obtained after placement of a CP shunt, revealing improvements in the cyst size and hygromas.

at the most recent follow-up visit, whereas $60 \%$ remained macrocephalic.

\section{Patients Presenting With Hydrocephalus}

In the current study, six patients (14\%) presented with hydrocephalus. Three of them had received a shunt before fenestration; these three patients remained shunt dependent following the initial fenestration. Two of them required subsequent fenestrations with concurrent VP shunt revisions. Two patients underwent primary fenestration for an arachnoid cyst and required subsequent placement of a VP shunt for persistent hydrocephalus. One of these patients had a posterior fossa cyst (Fig. 2), whereas another had a suprasellar cyst. One patient who had presented with hydrocephalus, macrocephaly, and a middle fossa arachnoid cyst that communicated extensively with the lateral ventricle demonstrated resolution of the cyst and hydrocephalus after fenestration, and remained shunt free until the most recent follow up.

\section{Patients With Other Presentations}

Fifteen patients presented with neither macrocephaly nor hydrocephalus. Of these patients, four had an arach-

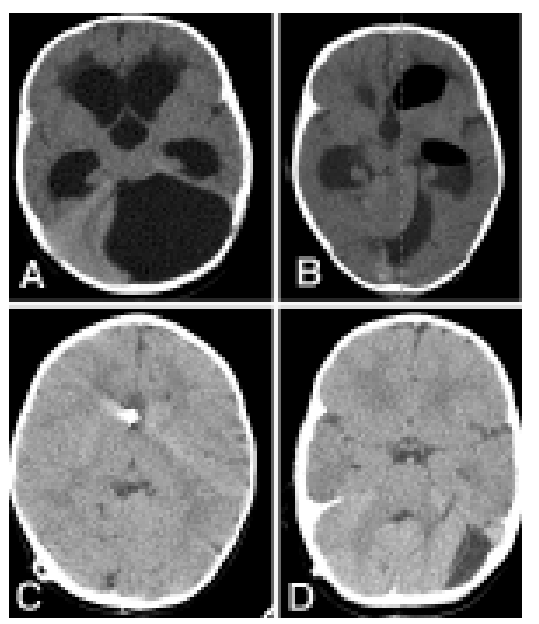

FIG. 2. A: Initial non-contrast-enhanced CT scan exhibiting a posterior fossa cyst and hydrocephalus. B: Postoperative CT scan obtained after fenestration showing improvement in the cyst size as well as ventriculomegaly. C and D: Follow-up CT scans revealing the patient's status following placement of a VP shunt. noid cyst that had been demonstrated on routine fetal ultrasonography. Six patients were incidentally diagnosed with arachnoid cysts as young children; these patients constituted a small percentage of all of those incidentally diagnosed with arachnoid cysts at our institution. Two patients presented with seizures. One patient each presented with motor deficits and developmental delay. Of these 15 patients, $14(93 \%)$ remained shunt free following fenestration for the arachnoid cyst. One patient, a 23-month-old girl with a concurrent intracranial dermoid cyst and middle fossa arachnoid cyst, required placement of a VP shunt 4 weeks after a fenestration procedure.

\section{Procedural Complications}

There were no perioperative deaths in any of the patients. Subacute subdural hematomas developed postoperatively in two patients $(5 \%)$. One occurred after fenestration of a posterior fossa cyst, and the other after fenestration of a middle fossa arachnoid cyst (Fig. 3). Further surgical intervention was not required in either case. Three patients $(7 \%)$ suffered subdural hygromas after fenestration. Two of these patients had middle fossa arachnoid cysts and required subsequent placement of a $\mathrm{CP}$ shunt. The third patient had a frontotemporal cyst along the convexity and did not require further treatment. Two patients had CSF leaks after fenestration, each requiring reoperation with temporary lumbar drainage. Pseudomeningoceles developed in three patients following fenestration; two patients required repeated surgery for dural repairs and a third underwent placement of a CP shunt. Meningitis developed in one patient after surgery and was successfully treated with antibiotics, without any clinical sequelae.

\section{Shunt Revisions}

Among the 18 patients with shunts, three received them before fenestration, and 15 received them after the fenestration procedure. Eleven patients underwent placement of a VP shunt after fenestration, whereas seven underwent placement of a $\mathrm{CP}$ shunt. Ten patients $(55 \%)$ required a total of 17 shunt revisions during the follow-up period following fenestration. Of the 11 patients with VP shunts, five $(45 \%)$ required at least one revision. Of the seven patients with CP shunts, five $(71 \%)$ required a subsequent revision.

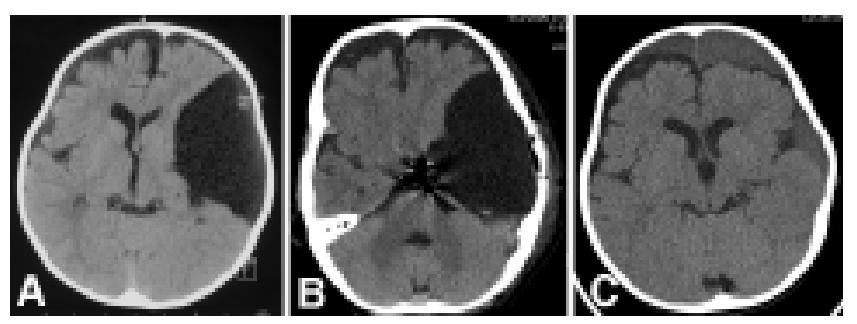

FIG. 3. A: Preoperative non-contrast-enhanced CT scan demonstrating a left middle fossa arachnoid cyst. B: A CT scan initially obtained after left craniotomy revealing fenestration into basal cisterns. C: A CT scan obtained at the 3-month follow up, showing a significant decrease in the size of the arachnoid cyst. Subdural hematomas developed in the frontotemporal region in this patient during the follow-up period. 


\section{Discussion}

Arachnoid cysts were initially described by Bright ${ }^{5}$ in 1831 as "serous cysts within the arachnoid" and have since been determined to constitute approximately $1 \%$ of all intracranial mass lesions. ${ }^{17}$ Many theories have been offered to explain the pathogenesis of arachnoid cysts, including inciting events such as infection, trauma, and metabolic alterations. ${ }^{17,22}$ It has been postulated that arachnoid cysts primarily result from duplication and splitting of the arachnoid endomeninx, which normally develops into the pia and arachnoid layers as well as the subarachnoid space. ${ }^{22}$ The potential expansion of arachnoid cysts during subsequent development likely has multifactorial causes. One theory has supported growth via an underlying CSF pressure gradient or unidirectional ball-valve mechanism..$^{10}$ Personnel at our institution have also reported that arachnoid cysts can expand due to an oncotic gradient resulting from increased protein concentrations within the cyst. ${ }^{24}$ Finally, data from some studies have shown that the membranes of arachnoid cysts harbor secretory properties that may be partially responsible for their potential to expand. ${ }^{9}$ In any case, progressive growth of arachnoid cysts may cause secondary regional effects such as hypoplasia of the temporal lobe as well as manifest as symptoms such as seizures, developmental delay,

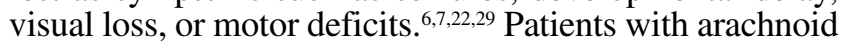
cysts may also present with hemorrhagic events, especially following head trauma. ${ }^{1,19,20,23}$ Younger patients $(<2$ years of age) are more likely to present with nonspecific macrocephaly than other symptoms or signs. ${ }^{7,15,17}$ In the current study, one half of all patients presented with macrocephaly without underlying ventriculomegaly.

It seems likely that there is a direct relationship between the development of an arachnoid cyst and abnormal CSF flow dynamics. The development and expansion of arachnoid cysts may be a form fruste of hydrocephalus. Some authors have previously described a similar correlation. Martinez-Lage et al. ${ }^{17}$ have reported that $60 \%$ of supratentorial and $80 \%$ of infratentorial arachnoid cysts have associated ventriculomegaly. ${ }^{17}$ Data from his same study also revealed that the expansion of arachnoid cysts over time may be an outlet or may provide a dampening effect from raised ICPs due to hydrocephalus. ${ }^{17}$

Considering the clinical presentation of patients with an arachnoid cyst may provide some knowledge regarding the type of treatment that likely will be required. Patients with intracranial arachnoid cysts presenting with nonspecific macrocephaly or ventriculomegaly are more likely to become shunt dependent than are those presenting with other manifestations, such as seizures or developmental delay. In the current study, infants presenting with macrocephaly alone were significantly more likely to require shunt placement compared with those presenting with other symptoms $(57 \%$ compared with $7 \%$; $\mathrm{p}$ value $=$ 0.0039 , two-tailed Fisher exact test). In previously published data from our institution, $73 \%$ of patients without ventriculomegaly remained shunt independent, compared with only $32 \%$ of patients with hydrocephalus. ${ }^{7}$ Data from another study demonstrated that $100 \%$ of patients with middle fossa arachnoid cysts who had presented with hydrocephalus required eventual shunt placement, compared with 55\% of those who had presented with nonspecific macrocephaly. ${ }^{15}$ It has been further suggested that the degree of communication between the cyst and the ventricular space remains another factor that predicts the requirement for further shunt placement. ${ }^{15,26}$

The optimal initial treatment for symptomatic or radiographically expanding cysts remains controversial. On one hand, craniotomy with fenestration of an arachnoid cyst is sufficient treatment in approximately $60 \%$ of patients, and the rate can be even higher in patients without hydrocephalus or macrocephaly.? When successful, fenestration confers the benefit of shunt independence. There are, however, well-described risks associated with craniotomy and fenestration. ${ }^{6-8,25}$ On the other hand, shunting procedures may also treat the underlying origins of impaired CSF dynamics and elevated pressure, and may offer a lower initial risk profile than fenestration., ${ }^{3,6,12,27}$ However, shunt procedures frequently require reoperation and are associated with the lifelong burden of potential shunt malfunction, shunt infection, and overdrainage. ${ }^{2,3,6,7,18}$ In the current study, $71 \%$ of patients with $\mathrm{CP}$ shunts and $45 \%$ of those with VP shunts required revision during the follow-up period.

\section{Conclusions}

In patients presenting with ventriculomegaly in conjunction with an arachnoid cyst, we recommend initial cyst fenestration and VP shunt placement in most cases. Better results have been demonstrated when VP shunt insertion precedes cyst fenestration. ${ }^{7,16}$ The precise role of endoscopic fenestration with the creation of a third ventriculostomy in such patients remains to be determined and yet is quite promising. In patients presenting with nonspecific macrocephaly in conjunction with an arachnoid cyst, we recommend initial cyst fenestration plus subsequent shunt placement as necessary. In our experience, more than $40 \%$ of these patients will remain shunt free. Finally, patients diagnosed due to other presentations should undergo fenestration alone and subsequent shunt placement only as necessary, given that fewer than $10 \%$ of these patients will require shunts.

\section{References}

1. Albuquerque FC, Giannotta SL: Arachnoid cyst rupture producing subdural hygroma and intracranial hypertension: case reports. Neurosurgery 41:951-956, 1997

2. Aoki N, Sakai T, Umezawa Y: Slit ventricle syndrome after cyst-peritoneal shunting for the treatment of intracranial arachnoid cyst. Childs Nerv Syst 6:41-43, 1990

3. Arai H, Sato K, Wachi A, Okuda O, Takeda N: Arachnoid cysts of the middle cranial fossa: experience with 77 patients who were treated with cystoperitoneal shunting. Neurosurgery 39: 1108-1113, 1996

4. Balsubramaniam C, Laurent J, Rouah E, Armstrong D, Feldstein N, Schneider S, et al: Congenital arachnoid cysts in children. Pediatr Neurosci 15:223-228, 1989

5. Bright R: Reports of Medical Cases Selected With a View of Illustrating the Symptoms and Cure of Diseases by a Reference to Morbid Anatomy, Vol 2. London: Longman, Rees, Orne, Brown and Green, 1827-1831

6. Ciricillo SF, Cogen PH, Harsh GR, Edwards MS: Intracranial arachnoid cysts in children. A comparison of the effects of fenestration and shunting. J Neurosurg 74:230-235, 1991

7. Fewel ME, Levy ML, McComb JG: Surgical treatment of 95 children with 102 intracranial arachnoid cysts. Pediatr Neurosurg 25:165-173, 1996 
8. Galassi E, Gaist G, Giuliani G, Pozzati E: Arachnoid cysts of the middle cranial fossa: experience with 77 cases treated surgically. Acta Neurochir Suppl (Wien) 42:201-204, 1988

9. Go KG, Houthoff HJ, Blaauw EH, Havinga P, Hartsuiker J: Arachnoid cysts of the sylvian fissure. Evidence of fluid secretion. J Neurosurg 60:803-813, 1984

10. Gosalakkal JA: Intracranial arachnoid cysts in children: a review of pathogenesis, clinical features, and management. Pediatr Neurol 26:93-98, 2002

11. Hanieh A, Simpson DA, North JB: Arachnoid cysts: a critical review of 41 cases. Childs Nerv Syst 4:92-96, 1988

12. Harsh GR IV, Edwards MS, Wilson CB: Intracranial arachnoid cysts in children. J Neurosurg 64:835-842, 1986

13. Kang JK, Lee KS, Lee IW, Jeun SS, Son BC, Jung CK, et al: Shunt-independent surgical treatment of middle cranial fossa arachnoid cysts in children. Childs Nerv Syst 16:111-116, 2000

14. Lange M, Oeckler R: Results of surgical treatment in patients with arachnoid cysts. Acta Neurochir (Wien) 87:99-104, 1987

15. Levy ML, Meltzer HS, Hughes S, Aryan HE, Yoo K, Amar AP: Hydrocephalus in children with middle fossa arachnoid cysts. J Neurosurg 101 (Suppl 1):25-31, 2004

16. Marinov M, Undjian S, Wetzka P: An evaluation of the surgical treatment of intracranial arachnoid cysts in children. Childs Nerv Syst 5:177-183, 1989

17. Martinez-Lage JF, Ruiz-Macia D, Valenti JA, Poza M: Development of a middle fossa arachnoid cyst. A theory on its pathogenesis. Childs Nerv Syst 15:94-97, 1999

18. Oberbauer RW, Haase J, Pucher R: Arachnoid cysts in children: a European co-operative study. Childs Nerv Syst 8:281-286, 1992

19. Page AC, Mohan D, Paxton RM: Arachnoid cysts of the middle fossa predispose to subdural haematoma formation fact or fiction? Acta Neurochir Suppl (Wien) 42:210-215, 1988

20. Parsch CS, Krauss J, Hofmann E, Meixensberger J, Roosen K: Arachnoid cysts associated with subdural hematomas and hygromas: analysis of 16 cases, long-term follow-up, and review of the literature. Neurosurgery 40:483-490, 1997

21. Raffel C, McComb JG: To shunt or to fenestrate: which is the best surgical treatment for arachnoid cysts in pediatric patients? Neurosurgery 23:338-342, 1988

22. Rengachary SS, Watanabe I, Brackett CE: Pathogenesis of intracranial arachnoid cysts. Surg Neurol 9:139-144, 1978

23. Robinson R: Congenital cysts of the brain: arachnoid malfomations. Prog Neurol Surg 4:133-174, 1971

24. Sandberg DI, McComb JG, Krieger MD: Chemical analysis of fluid obtained from intracranial arachnoid cysts in pediatric patients. J Neurosurg 103 (5 Suppl):427-432, 2005

25. Sato H, Sato N, Katayama S, Tamaki N, Matsumoto S: Effective shunt-independent treatment for primary middle fossa arachnoid cyst. Childs Nerv Syst 7:375-381, 1991

26. Shurtleff DB, Eliason BC, Oakland JA: Congenital brain cysts in infancy: diagnosis, treatment, and follow-up. Teratology 7:183-190, 1973

27. Stein SC: Intracranial developmental cysts in children: treatment by cystoperitoneal shunting. Neurosurgery 8:647-650, 1981

28. Villavicencio AT, Wellons JC III, George TM: Avoiding complicated shunt systems by open fenestration of symptomatic fourth ventricular cysts associated with hydrocephalus. Pediatr Neurosurg 29:314-319, 1998

29. Zaatreh MM, Bates ER, Hooper SR, Palmer G, Elmenshawi EE, Courvoisie HE, et al: Morphometric and neuropsychologic studies in children with arachnoid cysts. Pediatr Neurol 26: 134-138, 2002

Manuscript submitted December 5, 2006.

Accepted January 5, 2007.

Address reprint requests to: Gabriel Zada, M.D., Department of Neurosurgery, University of Southern California, 1200 North State Street, Suite 5046, Los Angeles, California 90089. email: gzada@usc.edu. 